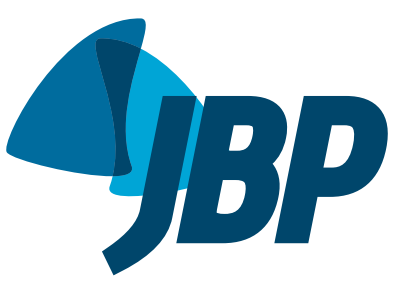

\section{An uncommon chest mass: oleothorax}

\author{
Bruno Hochhegger ${ }^{1}$, Gláucia Zanetti², Edson Marchiori
}

\section{TO THE EDITOR:}

We would like to report a case of unilateral oleothorax, which generated great diagnostic difficulties and was initially interpreted as a lung mass, probably of neoplastic origin. An 84-year-old woman presented with a dry cough that had persisted for four months. A chest $X$-ray showed a mass in the right hemithorax (Figure $1 \mathrm{~A}$ ). Due to the suspicion of lung cancer, the patient underwent $\mathrm{CT}$, and

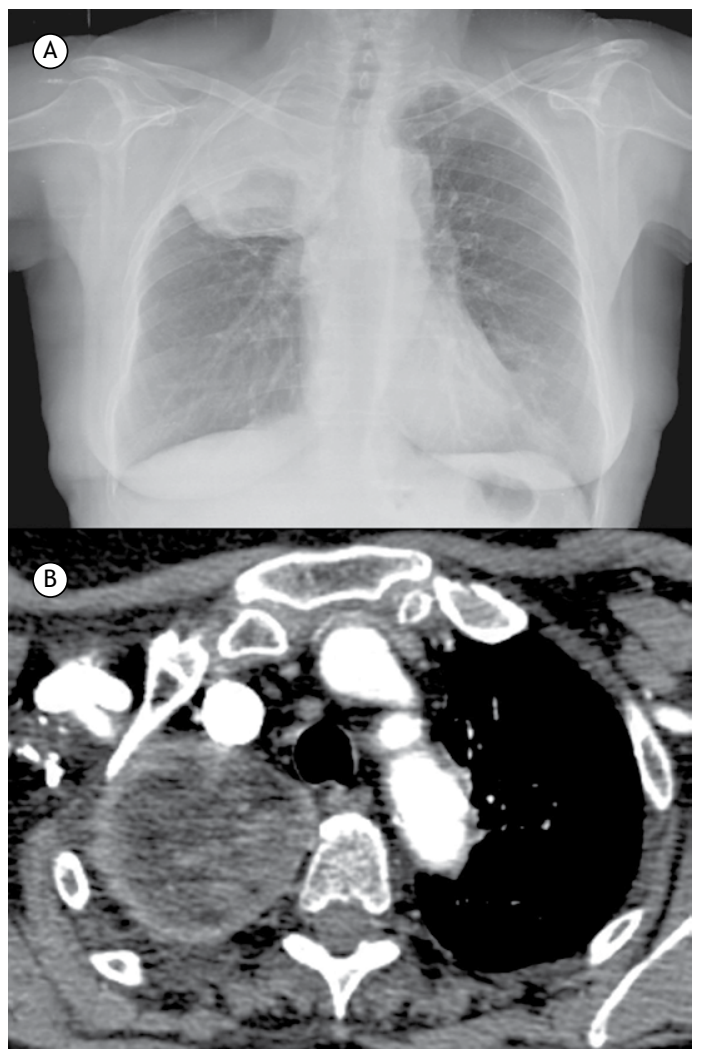

Figure 1. In $A$, a chest $X$-ray showing a mass in the apical region of the right hemithorax. In $B$, an axial $C T$ scan demonstrating that the mass was heterogeneous and well delimited, with no evidence of parietal invasion. the scans demonstrated a posteriorly heterogeneous mass in the upper right hemithorax (Figure 1B). Aspirated viscous pleural fluid proved to be mineral oil. Upon further discussion, the patient vaguely remembered that she had been treated for tuberculosis and had received an oil injection 60 years prior.

Oleothorax, the intrapleural or extrapleural instillation of mineral or vegetable oil into the pleural space, was widely used from the early decades of the 20th century until the late 1940s. Oleothorax was a form of collapse therapy used to inhibit the multiplication and dissemination of pulmonary Mycobacterium tuberculosis. In addition to exerting a mass effect on the adjacent lung, such substances were caustic and produced obliterative pleuritis, which justified their use in tuberculous empyemas. The amount of oil used varied widely $(100-2,000 \mathrm{~mL})$. The recommended length of therapy was up to 2 years, with removal of the oil thereafter. ${ }^{(1-5)}$ However, asymptomatic patients were often lost to follow-up, and the oil was never removed, as in our case.

Failure to drain the oil has been associated with diverse complications, some occurring decades later. Long-term complications of oleothorax include bronchopleural fistula, pleurocutaneous fistula, airway obstruction, recurrent tuberculous empyema, chest wall abscess, respiratory distress from an expanding mass, among others. Extrapleural oleothorax produced fewer complications than did the pleural variety. With the advent of effective antituberculosis chemotherapy, the technique was largely abandoned in the 1950s. ${ }^{(2-5)}$ The most characteristic CT finding of oleothorax is an encapsulated pleural collection with three levels: a superior level with air, an intermediary level with lipid content ( -30 to -150 Hounsfield units), and an inferior level with positive densities. ${ }^{(3-5)}$

A diagnosis of oleothorax might be achieved from CT images, and the recognition of this pattern is important because the patient might not know or remember the details of the antecedent intervention performed many years prior. In summary, oleothorax should be included in the differential diagnosis of thoracic masses, particularly in elderly patients.

\title{
REFERENCES
}

- López Riolobos C, Zamora García E, García Castillo E. Bilateral Oleothorax. Arch Bronconeumol. 2016;52(4):218. http://dx.doi. org/10.1016/j.arbres.2014.12.013

2. Hutton L. Oleothorax: expanding pleural lesion. AJR Am J Roentgenol. 1984;142(6):1107-10. http://dx.doi.org/10.2214/ajr.142.6.1107

3. Hochhegger B, Zanetti G, Marchiori E. Oleothorax simulating pulmonary neoplasm. Ann Thorac Surg. 2013;95(5):1807. http://dx.doi. org/10.1016/j.athoracsur.2012.09.076
4. Fahy RJ, Morales J, King M. Late reactivation of tuberculosis in an oleothorax. J Thorac Imaging. 2004;19(1):35-7. http://dx.doi. org/10.1097/00005382-200401000-00006

5. Freedman BJ, McCarthy DM, Feldman F, Feirt N. Fatty infiltration of osseous structures: a long-term complication of oleothorax-case report. Radiology. 1999;210(2):515-7. http://dx.doi.org/10.1148/ radiology.210.2.r99fe42515 